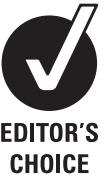

CHOICE
- Additional materials are published online only. To view these files please visit the journal online (http://jmg.bmj. com/content/49/2.toc).

1 Department of Neurology and Center for Translational Medicine, Peking Union Medical College Hospital, Chinese Academy of Medical Sciences \& Peking Union Medical College (CAMS \& PUMC), Beijing, China ${ }^{2}$ State Key Laboratory of Medical Molecular Biology, Institute of Basic Medical Sciences, CAMS \& PUMC, Beijing, China

\section{Correspondence to}

Professor Xue Zhang, State Key Laboratory of Medical Molecular Biology, Institute of Basic Medical Sciences, Chinese Academy of Medical Sciences \& Peking Union Medical College, 5 Dong Dan San Tiao, Beijing, China; xuezhang@pumc.edu.cn

Professor Li-Ying Cui, Department of Neurology, Peking Union Medical College Hospital, Chinese Academy of Medical Sciences \& Peking Union Medical College, 1 Shuaifuyuan, Beiijing, China; pumchcly@yahoo.com.cn

Received 25 November 2011 Accepted 13 December 2011 Published Online First 29 December 2011

\title{
Mutations in PRRT2 result in paroxysmal dyskinesias with marked variability in clinical expression
}

\author{
Oing Liu, ${ }^{1}$ Zhan $\mathrm{Oi}^{2}{ }^{2}$ Xin-Hua Wan, ${ }^{1}$ Jing-Yun Li, ${ }^{2}$ Lei Shi, ${ }^{2}$ Oiang Lu, ${ }^{1}$ \\ Xiang-Oin Zhou, ${ }^{1}$ Lei Oiao, ${ }^{1}$ Li-Wen Wu, ${ }^{1}$ Xiu-Oin Liu, ${ }^{1}$ Wei Yang, ${ }^{2}$ Ying Liu, ${ }^{2}$ \\ Li-Ying Cui, ${ }^{1}$ Xue Zhang ${ }^{1,2}$
}

\begin{abstract}
Background Paroxysmal dyskinesias (PDs), a clinically and genetically heterogeneous group of episodic movement disorders, include kinesigenic PD (PKD), exercise-induced PD (PED) and non-kinesigenic PD (PNKD). These disorders are all transmitted as autosomal dominant traits with incomplete penetrance. Several PDrelated genetic disorders, including PKD and familial infantile convulsions with paroxysmal choreoathetosis (ICCA), mapped to the same region on chromosome 16 . Independent genetic studies have recently revealed that PKD can be caused by loss-of-function mutations in the proline-rich transmembrane protein 2 gene (PRRT2). We tested the hypothesis that other forms of PDs are also due to PRRT2 mutations.
\end{abstract}

Methods/results The whole genomic region of PRRT2 was sequenced in six Han Chinese families and 15 sporadic cases of PD-related phenotypes. The previously reported mutation, c.649dupC (p.R217Pfs*7), was found in two families with PKD, one family with ICCA, one family with PNKD-like phenotype, and two sporadic cases with PED. In an additional ICCA family, a novel frameshift mutation, c.904dupG (p.D302Gfs*38), was identified. A missense mutation, c.913G $\rightarrow$ A (p.G305R), and a synonymous substitution, c.1011C $\rightarrow$ T (p.G337G), were also detected in two sporadic PKD cases.

Conclusion This study shows that PKD, ICCA and some other PD-related phenotypes are part of the same phenotypic spectrum, caused by mutations in PRRT2. This underscores the complexity of the phenotypic consequences of PRRT2 mutations.

\section{INTRODUCTION}

Paroxysmal dyskinesias (PDs) are a group of disorders characterised by episodes of involuntary movements that correspond to attacks of choreoathetosis or dystonia. They can be classified into three main categories: paroxysmal kinesigenic dyskinesia (PKD), paroxysmal exercise-induced dyskinesia (PED) and paroxysmal non-kinesigenic dyskinesia (PNKD). ${ }^{1}$ PKD (OMIM 128200), also known as paroxysmal kinesigenic choreoathetosis or episodic kinesigenic dyskinesia 1, represents the most common type of $\mathrm{PD}$, presenting with recurrent and brief attacks of dystonia and choreoathetosis. The diagnostic criteria for idiopathic $\mathrm{PKD}^{2}$ includes sudden movements as the trigger, a short duration of attacks ( $<1 \mathrm{~min}$ ), lack of loss of consciousness or pain during attacks, a good response to antiepileptic drug treatment, exclusion of other organic diseases, and an onset age between 1 and 20 years. In contrast with PKD, PED is a neurological symptom with wide phenotypic variability and is usually triggered by prolonged physical exertion. ${ }^{3}$ PNKD (OMIM 118800) would not be precipitated by sudden movements, but alcohol, caffeine, stress, sleep deprivation and exercise can lower the threshold of attacks. ${ }^{4}$ Mutations in the myofibrillogenesis regulator-1 gene $(M R 1)$ have been revealed in several PNKD families. $^{5-7}$

PD may be variably associated with epilepsy. ${ }^{8}$ Benign familial infantile seizures (BFIS1, OMIM 601764; BFIS2, OMIM 605751), also called benign familial infantile convulsions, is mostly mentioned, as it can occur independently of or concurrently with PD. ${ }^{9-11}$ BFIS is an autosomal dominant disorder described as afebrile seizures occurring between 3 and 12 months of age, with good response to medication and no neurological sequelae. ${ }^{12}$ Familial infantile convulsions with paroxysmal choreoathetosis (ICCA, OMIM 602066) shares overlapping clinical features with BFIS and PKD. ${ }^{11}{ }^{13}$ Epilepsy may also coexist with PED. ${ }^{14}$ Autosomal recessive rolandic epilepsy with PED and writer's cramp (RE-PED-WC, OMIM 608105) shows phenotypic similarities to ICCA. $^{15}$ Very interestingly, PKD, BFIS2, ICCA and RE-PED-WC all map to the same pericentromeric region on chromosome 16 , suggesting that they are probably allelic disorders. ${ }^{8} 1213$ 15-17

Two Chinese groups have recently identified PRRT2, encoding proline-rich transmembrane protein 2, as a causative gene for PKD. ${ }^{18}{ }^{19}$ We thus performed mutation screening of PRRT2 in our collected families and sporadic cases affected with PKD or other PD-related phenotypes.

\section{SUBJECTS AND METHODS}

We ascertained six Han Chinese families (see online supplementary figure 1 for pedigrees) and 15 sporadic cases of PKD, ICCA, PED or PNKDlike phenotypes (details in online supplementary table 1).

Clinical findings in affected individuals in families $\mathrm{A}$ and $\mathrm{B}$ are consistent with a diagnosis of ICCA. Individuals were considered as affected if they had either BFIS or PD, or if they had both a history of BFIS and PD. Nine affected individuals in family A were available for clinical evaluation. The traits of BFIS and PD in the family were 
unconnected, with six and three individuals having isolated BFIS and PD, respectively. Nearly all cases of BFIS manifested as deviation of eyes to one side, an altered consciousness, and bilateral limb jerks with hypertonia. The seizures occurred mostly when awake and were isolated except that, in two cases (IV-7 and V-1), seizures happened during sleep and were clustered. None of the interictal EEGs showed epileptiform abnormalities between attacks, and CT scanning or MRI were normal in the cases examined. Both of the two affected individuals in family B had BFIS and PD. Notably, the characteristics of choreoathetosis in these two families were consistent with PED. The age of onset was $8-10$ years, and remission seemed to be around 20 years. Choreoathetosis was of the dystonic type, occurring at rest, or could be provoked by anxiety, fatigue or exercise after a few seconds. The limbs of one side were affected, with short duration of attacks lasting $<5 \mathrm{~min}$. Response to antiepileptic drugs varied in both families.

Affected individuals in family $\mathrm{C}$ had a PNKD-like phenotype. The trunk and limbs on both sides were involved, with a long duration. The onset age of PD was about 7-8 years, except in the proband (IV-2), where the age was as young as 2 years with seemingly more severe symptoms. The age of remission was quite different, ranging from 2 to over 50. Alcohol, anxiety and fatigue were described as precipitants by affected individuals. Drinking alcohol was a definite trigger for III-5. Attacks could occur as often as 10-20 times per day. III-5 and IV-2 of family C described an obvious benefit of sleep during episodes. The anticonvulsive drugs were not effective as suggested before. ${ }^{4}$ The proband also had migraine. III-7, in particular, showed a typical generalised tonic-clonic type of seizure with normal EEG. The epilepsy began at the age of 20 with automatic remission in 2 years. Unfortunately, she, as well as II-2 and III-1, refused to participate in our genetic studies. No history of central nervous system disease or damage was found in any of the participating patients, and psychomotor development was normal on neurological examination.

In families D-F, clinical presentations of most affected individuals met the diagnostic criteria for PKD in all aspects. ${ }^{1}$ Among the 15 sporadic cases, 10 had PKD (cases 1, 2, 4, 5, 6, 8, 9, 10, 12 and 13), four had PED (cases 3,11, 14 and 15), and one had PNKD-like phenotype (case 7) (online supplementary table 1).

After obtaining informed consent, we collected peripheral blood samples for DNA from 11 individuals (six affected and five unaffected) in family $\mathrm{A}$, the probands of families $\mathrm{B}-\mathrm{F}$, and all the 15 sporadic cases. For mutation screening of the PRRT2 gene, we PCR-amplified the entire gene into four overlapping fragments and sequenced them directly after purification. ${ }^{19}$ We also

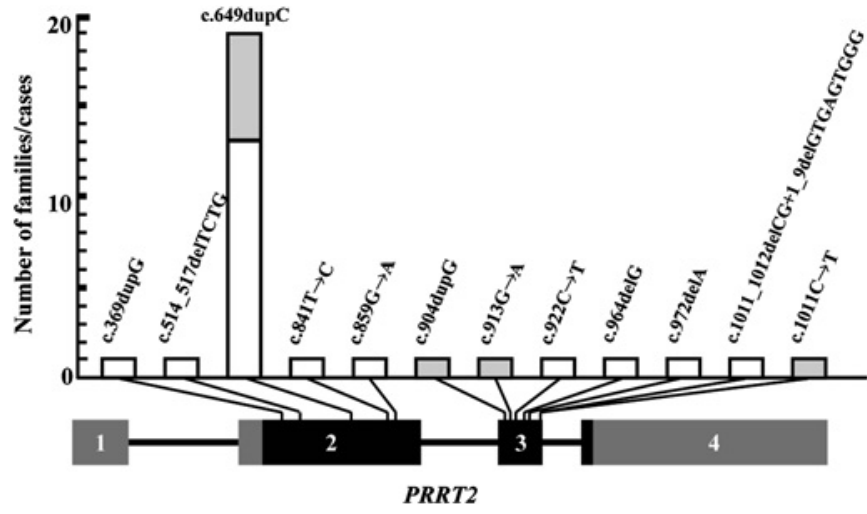

Figure 1 Gene structure of PRRT2 with a summary of identified mutations in paroxysmal kinesigenic dyskinesia, infantile convulsions with paroxysmal choreoathetosis, and other paroxysmal dyskinesiarelated phenotypes. For each identified mutation, its relative position in the PRRT2 gene and the numbers of families/cases detected with the mutation are indicated. Light-grey bars represent mutations reported in the present study.

performed genomic sequencing of PRRT2 in 192 unrelated Han Chinese controls.

\section{RESULTS}

We sequenced PRRT2 in one affected individual per family and identified the reported c.649dupC (p.R217Pfs*7) mutation ${ }^{18} 19$ in families A, C, E and F (figure 1 and table 1). This mutation was detected in all available affected individuals of family A. In family B, we found a novel single base duplication (c.904dupG), producing a frameshift and premature termination codon (p.D302Gfs*38) (figure 1, table 1 and online supplementary figure 2). Sequence analysis of PRRT2 in sporadic cases identified the c.649dupC (p.R217Pfs*7) mutation in two cases with PED, and a novel missense mutation, $c .913 \mathrm{G} \rightarrow \mathrm{A}$, in one with $\mathrm{PKD}$ (figure 1, table 1 and online supplementary figure 2). This missense mutation replaces a non-polar glycine $(G)$ (online supplementary figure 3) with a basic arginine (R) at amino acid residue 305 (p.G305R). We also found a synonymous substitution, c.1011C $\rightarrow \mathrm{T}$ (p.G337G), in a sporadic case with PKD. None of the identified mutations was found in the 192 controls. Consistent with the other two parallel reports on $\mathrm{PKD},{ }^{18} 19$ the mutations involved loss of function and suggest haploinsufficiency as the molecular mechanism.

To determine whether the PNKD-like phenotype in family $\mathrm{C}$ was caused by a mutation in $M R 1$, we also sequenced this gene and found no pathogenic mutations (data not shown).

Table 1 Major clinical features in families/cases with identified PRRT2 mutations

\begin{tabular}{|c|c|c|c|c|c|}
\hline Subject & Main phenotype & Accompanied features & Trigger of PD & Mutation DNA & Amino acid \\
\hline Family A & ICCA & - & $A x, E x$ & c.649dupC & p.R217Pfs*7 \\
\hline Family B & ICCA & - & $A x, E x$ & c.904dupG & p.D302Gfs*38 \\
\hline Family C & PNKD-like & Migraine & $\mathrm{Ah}, \mathrm{Ft}, \mathrm{Ax}$ & c.649dupC & p.R217Pfs*7 \\
\hline Family E & PKD & - & $\mathrm{Sm}$ & c.649dupC & p.R217Pfs ${ }^{*} 7$ \\
\hline Family F & PKD & Migraine, FC & $\mathrm{Sm}$ & c.649dupC & p.R217Pfs*7 \\
\hline Case 5 & PKD & FC & $\mathrm{Sm}$ & c. $1011 \mathrm{C} \rightarrow \mathrm{T}$ & p.G337G \\
\hline Case 13 & PKD & - & Sm & c. $913 \mathrm{G} \rightarrow \mathrm{A}$ & p.G305R \\
\hline Case 14 & PED & - & Ex & c.649dupC & p.R217Pfs*7 \\
\hline Case 15 & PED & - & Ex, St & c.649dupC & p.R217Pfs*7 \\
\hline
\end{tabular}

$\mathrm{Ax}$, anxiety; Ex, exercise; FC, febrile convulsions; Ft, fatigue; ICCA, infantile convulsions with paroxysmal choreoathetosis; PD, paroxysmal dyskinesia; PED, paroxysmal exercise-induced dyskinesia; PKD, paroxysmal kinesigenic dyskinesia; PNKD, paroxysmal non-kinesigenic dyskinesia; Sm, sudden movement; St, startle. 


\section{DISCUSSION}

With the use of next-generation sequencing, two Chinese groups have recently identified PRRT2 as a causative gene for PKD. ${ }^{18} 19$ Chen and colleagues identified loss-of-function PRRT2 mutations in all their eight study families. ${ }^{18}$ In three of their four study families, Li and colleagues found PRRT2 mutations, including two frameshifts and one missense. ${ }^{19}$ They also showed that PRRT2 was mutated in approximately one-third of the sporadic PKD cases (10/29). ${ }^{19}$ In the present study, we sequenced the PRRT2 gene in three familial and 10 sporadic $P K D$ cases, and detected the previously reported mutation, c.649dupC (p.R217Pfs*7), ${ }^{18}{ }^{19}$ in two families, and a novel missense mutation in a sporadic case (table 1). Taken together, these results indicate that $P R R T 2$ mutations are responsible for most familial PKD and only a subset of sporadic PKD, and suggest the presence of genetic heterogeneity in PKD.

Previous genetic linkage studies in multiple families had suggested that BFIS2, ICCA and RE-PED-WC may be allelic with PKD. ${ }^{8} 1213^{15-17}$ We have found PRRT2 mutations in two Chinese families with ICCA, indicating that ICCA is indeed part of the same phenotypic spectrum caused by mutations in PRRT2. It is conceivable that PRRT2 mutations are also responsible for BFIS2 and RE-PED-WC. We unexpectedly identified PRRT2 mutations in a Chinese family with a PNKD-like phenotype and in two sporadic cases of PED (table 1), further expanding the phenotypic spectrum caused by mutations in the same PRRT2 gene and underscoring the complexity of the phenotypic consequences of PRRT2 mutations. Our work, together with the previous genetic studies, ${ }^{8} 121315-17$ provides genetic evidence that PKD, ICCA and other PD-related phenotypes may represent a phenotypic continuum.

We and others ${ }^{18} 19$ have together identified 12 distinct PRRT2 mutations in familial and sporadic cases of PKD, ICCA or other PD-related phenotypes (figure 1). No obvious genotype-phenotype correlation was observed. Most strikingly, the c.649dupC (p.R217Pfs*7) mutation was detected in 19 of the 30 cases with PRRT2 mutations (16 familial and 14 sporadic cases) (figure 1). We later typed a CA repeat sequence $139 \mathrm{~kb}$ centromeric to PRRT2 in our familial and sporadic cases with the c.649dupC mutation (table 1$)^{19}$ and found that six sporadic cases lacked the allele linked to the mutation in family A (data not shown). These facts, together with the haplotyping data of Chen and colleagues, ${ }^{18}$ suggest that the cytosine (C) tract at nucleotides 641-649 of the PRRT2 cDNA (5'-CCCCCCCCC-3') may be the hotspot for a single $\mathrm{C}$ duplication. Further haplotype analysis is still needed to exclude the possibility of a founder effect.

Frequent detection of truncating PRRT2 mutations has suggested haploinsufficiency as the underlying mechanism. ${ }^{18} 19$ $\mathrm{Li}$ and colleagues also found three $\mathrm{PKD}$-causing missense mutations in PRRT2. ${ }^{19}$ The c.913G $\rightarrow$ A (p.G305R) missense mutation detected in the present study (figure 1 and table 1) changed a highly conserved amino acid residue (online supplementary figure 3). Assessment using PolyPhen-2 (http:// genetics.bwh.harvard.edu/pph2/) predicted a damaging effect of this mutation. The synonymous c.1011C $\rightarrow T$ (p.G337G) is located at a position very close to the exon-intron junction (figure 1), suggesting that it may affect splicing. Neither of the two substitutions was detected in our ethnically matched controls. At present, the pathogenicity of c.1011C $\rightarrow \mathrm{T}$ (p.G337G) lacks supportive evidence.

In summary, we found PRRT2 mutations in 38.1\% (9/21) of our study cases (six family probands and 15 sporadic) and demonstrated that PKD, ICCA and other PD-related phenotypes may be caused by mutation in the same PRRT2 gene. Further molecular studies would provide insight into the connection between epilepsy and paroxysmal movement disorders.

Acknowledgements We thank all individuals who participated in this study.

Funding This work was supported by the National Natural Science Foundation of China to QLiu (grant number 81100969), the Program for Changjiang Scholars and Innovative Research Team in University (PCSIRT) to XZ (grant number IRT1006) and a PUMCH fund to OLiu.

\section{Competing interests None.}

Patient consent Obtained.

Ethics approval This study was approved by the ethics committee of Peking Union Medical College Hospital, Chinese Academy of Medical Sciences.

Contributors QLiu, LYC and XZ analysed all data and wrote the paper. QLiu, ZQ, JYL, LS, WY and YL generated the experimental data, and QLiu, XHW, QLu, XOZ, LQ, LWW and XOL provided all clinical findings and patient samples.

Provenance and peer review Not commissioned; externally peer reviewed.

Data sharing statement Primer sequences not given in the text are available upon request.

\section{REFERENCES}

1. Jankovic J, Demirkiran M. Classification of paroxysmal dyskinesias and ataxias. Adv Neurol 2002;89:387-400.

2. Bruno MK, Hallett M, Gwinn-Hardy K, Sorensen B, Considine E, Tucker S, Lynch DR, Mathews KD, Swoboda KJ, Harris J, Soong BW, Ashizawa T, Jankovic J, Renner D, Fu YH, Ptacek LJ. Clinical evaluation of idiopathic paroxysmal kinesigenic dyskinesia: new diagnostic criteria. Neurology 2004;63:2280-7.

3. Demirkiran M, Jankovic J. Paroxysmal dyskinesias: clinical features and classification. Ann Neurol 1995;38:571-9.

4. Lance JW. Familial paroxysmal dystonic choreoathetosis and its differentiation from related syndromes. Ann Neurol 1977:2:285-93.

5. Rainier S, Thomas D, Tokarz D, Ming L, Bui M, Plein E, Zhao X, Lemons R, Albin R, Delaney C, Alvarado D, Fink JK. Myofibrillogenesis regulator 1 gene mutations cause paroxysmal dystonic choreoathetosis. Arch Neurol 2004;61:1025-9.

6. Lee HY, Xu Y, Huang Y, Ahn AH, Auburger GW, Pandolfo M, Kwiecinski H, Grimes $D A$, Lang AE, Nielsen JE, Averyanov $Y$, Servidei S, Friedman A, Van Bogaert P, Abramowicz MJ, Bruno MK, Sorensen BF, Tang L, Fu YH, Ptácek LJ. The gene for paroxysmal non-kinesigenic dyskinesia encodes an enzyme in a stress response pathway. Hum Mol Genet 2004;13:3161-70.

7. Chen DH, Matsushita M, Rainier S, Meaney B, Tisch L, Feleke A, Wolff J, Lipe H, Fink J, Bird TD, Raskind WH. Presence of alanine-to-valine substitutions in myofibrillogenesis regulator 1 in paroxysmal nonkinesigenic dyskinesia: confirmation in 2 kindreds. Arch Neurol 2005;62:597-600.

8. Rochette J, Roll P, Szepetowski P. Genetics of infantile seizures with paroxysmal dyskinesia: the infantile convulsions and choreoathetosis (ICCA) and ICCA-related syndromes. J Med Genet 2008;45:773-9.

9. Fukuyama Y, Okada R. Hereditary kinaesthetic reflex epilepsy. Report of five pedigrees with seizures induced by movement and review of literature. Proc Aust Assoc Neurol 1968;5:583-7.

10. Hudgins RL, Corbin KB. An uncommon seizure disorder: familial paroxysmal choreoathetosis. Brain 1966;89:199-204.

11. Szepetowski P, Rochette J, Berquin P, Piussan C, Lathrop GM, Monaco AP. Familia infantile convulsions and paroxysmal choreoathetosis: a new neurological syndrome linked to the pericentromeric region of human chromosome 16. Am J Hum Genet 1997;61:889-98.

12. Caraballo R, Pavek S, Lemainque A, Gastaldi M, Echenne B, Motte J, Genton P, Cersósimo R, Humbertclaude V, Fejerman N, Monaco AP, Lathrop MG, Rochette J, Szepetowski P. Linkage of benign familial infantile convulsions to chromosome 16p12-q12 suggests allelism to the infantile convulsions and choreoathetosis syndrome. Am J Hum Genet 2001;68:788-94

13. Swoboda KJ, Soong B, McKenna C, Brunt ER, Litt M, Bale JF Jr, Ashizawa T, Bennett LB, Bowcock AM, Roach ES, Gerson D, Matsuura T, Heydemann PT, Nespeca MP, Jankovic J, Leppert M, Ptácek LJ. Paroxysmal kinesigenic dyskinesia and infantile convulsions: clinical and linkage studies. Neurology 2000;55:224-30.

14. Brockmann K. The expanding phenotype of GLUT1-deficiency syndrome. Brain Dev 2009;31:545-52.

15. Guerrini R, Bonanni P, Nardocci N, Parmeggiani L, Piccirilli M, De Fusco M, Aridon P Ballabio A, Carrozzo R, Casari G. Autosomal recessive rolandic epilepsy with paroxysmal exercise-induced dystonia and writer's cramp: delineation of the syndrome and gene mapping to chromosome 16p12-11.2. Ann Neurol 1999:45:344-52

16. Tomita H, Nagamitsu S, Wakui K, Fukushima Y, Yamada K, Sadamatsu M, Masui A Konishi T, Matsuishi T, Aihara M, Shimizu K, Hashimoto K, Mineta M, Matsushima M, Tsujita T, Saito M, Tanaka H, Tsuji S, Takagi T, Nakamura Y, Nanko S, Kato N, 
Nakane Y, Niikawa N. Paroxysmal kinesigenic choreoathetosis locus maps to chromosome 16p11.2-q12.1. Am J Hum Genet 1999;65:1688-97.

17. Bennett LB, Roach ES, Bowcock AM. A locus for paroxysmal kinesigenic dyskinesia maps to human chromosome 16. Neurology 2000;54:125-30.

18. Chen WJ, Lin Y, Xiong ZQ, Wei W, Ni W, Tan GH, Guo SL, He J, Chen YF, Zhang QJ, Li HF, Lin Y, Murong SX, Xu J, Wang N, Wu ZY. Exome sequencing identifies truncating mutations in PRRT2 that cause paroxysmal kinesigenic dyskinesia. Nat Genet 2011;43:1252-5

19. Li J, Zhu X, Wang X, Sun W, Feng B, Du T, Sun B, Niu F, Wei H, Wu X, Dong L, Li L, Cai $X$, Wang $Y$, Liu $Y$. Targeted genomic sequencing identifies PRRT2 mutations as a cause of paroxysmal kinesigenic choreoathetosis. J Med Genet 2012:49:76-8

\section{DIFFERENTIAL DIAGNOSIS}

\section{Trustworthy guidance on your iPhone}
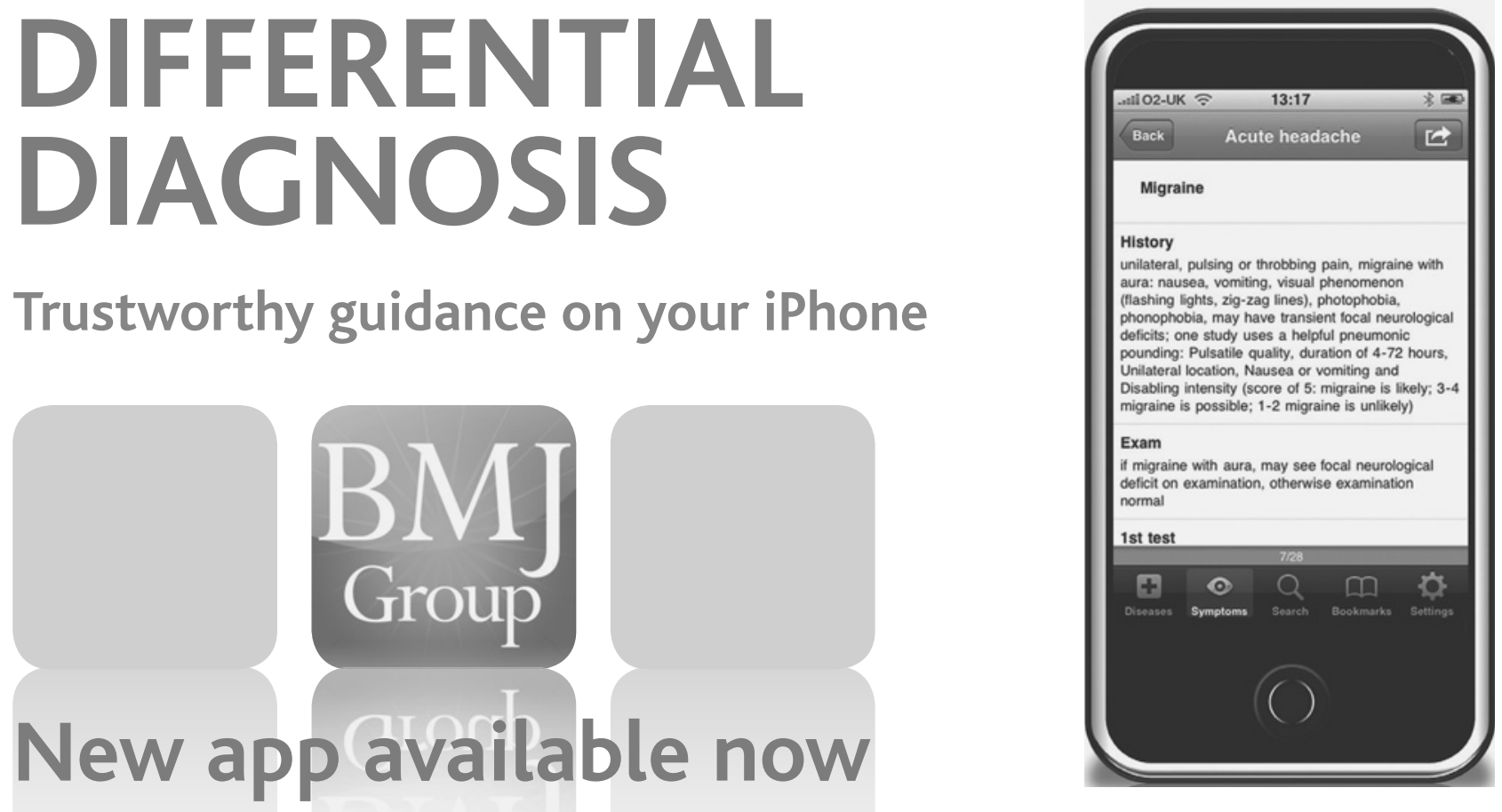

Find out more at bestpractice.bmj.com/differentials 